\title{
On the transition from initial data to travelling waves in the Fisher-KPP equation
}

\section{Jonathan A. Sherratt}

Department of Mathematics, Heriot-Watt University, Edinburgh EH14 4AS, UK and Mathematics Institute, University of Warwick, Coventry CV4 7AL, UK

Abstract. The Fisher-KPP equation $\mathrm{u}_{\mathrm{t}}=\mathrm{u}_{\mathrm{xx}}+\mathrm{u}(1-\mathrm{u})$ has a travelling wave solution for all speeds $\geqslant 2$. Initial data that decrease monotonically from 1 to 0 on $-\infty<\mathrm{x}<\infty$, with $\mathrm{u}(\mathrm{x}, 0)=\mathrm{O}_{\mathrm{s}}\left(e^{-\xi \mathrm{x}}\right)$ as $\mathrm{x} \rightarrow \infty$, are known to evolve to a travelling wave, whose speed depends on $\xi$. Here, it is shown that the relationship between wave speed and $\xi$ can be recovered by linearizing the Fisher-KPP equation about $\mathrm{u}=0$ and explicitly solving the linear equation. Moreover, the calculation predicts that in the case $\xi>1$, the solution for $\mathrm{u}_{\mathrm{x}} / \mathrm{u}$ itself evolves to a transition wave, moving ahead of the (minimum speed) $\mathrm{u}$ wave at the greater speed of $2 \xi$. Behind this transition, $\mathrm{u}_{\mathrm{x}} / \mathrm{u}=-\mathrm{x} /(2 \mathrm{t})$, while ahead of $i t$, $\mathrm{u}_{\mathrm{x}} / \mathrm{u}=-\xi$. The paper goes on to discuss the potential applications of the method to systems of coupled reaction-diffusion equations.

\section{Introduction}

Travelling wave fronts are an important and much studied solution form for reaction-diffusion equations, with important applications to chemistry, biology and medicine (see Needham et al. (1994), Okubo et al. (1989) and Orme \& Chaplain (1996) for examples and Murray (1989) for a review). Such solutions were first studied in the 1930s by Fisher (1937) and Kolmogoroff et al. (1937), for the scalar equation

$$
\frac{\partial u}{\partial t}=\frac{\partial^{2} u}{\partial x^{2}}+u(1-u)
$$

which is now known as the Fisher-KPP equation. This is important both as a generic example of a wide class of reaction-diffusion equations, and as a simple model for a range of biological phenomena. The original application of (1) by Fisher (1937) was to population genetics, with $u$ denoting the density of an advantageous gene, and the travelling wave solutions representing the spread of this gene through space. Subsequent applications have been very varied, including ecology, modelling waves of an invading population (Holmes et al., 1994) and

E-mail: jas@ma.hw.ac.uk. 
wound healing, with solutions representing healing waves of cells in the skin (Sherratt \& Murray, 1990).

Analysis of travelling wave solutions of (1) dates back to Kolmogoroff et al. (1937), who showed that travelling wave solutions, i.e. solutions that are functions of $x$-at, exist for all values of the wave speed $a \geqslant 2$; these waves are monotonically decreasing in $x$, approaching 1 as $x \rightarrow-\infty$ and 0 as $x \rightarrow+\infty$. There are corresponding monotonically increasing waves with negative speeds. In a ground-breaking piece of analysis, Kolmogoroff et al. (1937) also showed that the travelling wave with minimum speed 2 is the long time limit for any initial conditions satisfying $u \equiv 1$ for sufficiently large negative $x$, and $u \equiv 0$ for sufficiently large positive $x$.

In the 1970 s, a number of authors studied types of initial data that can evolve to waves travelling faster than the minimum speed. Independent use of the theory of branching processes for Brownian motion and traditional differential equation methods showed that initial conditions satisfying $u \rightarrow 1$ as $x \rightarrow-\infty$ and $u=O_{s}\left(\mathrm{e}^{-\xi x}\right)$ as $x \rightarrow+\infty$ evolve to the travelling wave of speed

$$
c(\xi)= \begin{cases}2, & \text { if } \xi \geqslant 1 \\ \xi+1 / \xi, & \text { if } \xi \leqslant 1\end{cases}
$$

(Larson, 1978; McKean, 1975; Rothe, 1978). Recently, this result has been extended by Booty et al. (1993), to show that when the exponential decay rate $\xi$ varies slowly in space, the wave speed adjusts to accommodate this variation.

In this paper, the insight that can be gained about this wave speed selection by linearizing (1) about $u=0$ and solving using Laplace transforms will be considered. The method of Laplace transforms has been used previously by Sleeman and Tuma (1984) to solve a piecewise linear version of the Fisher-KPP equation. They derive exact solutions, which converge to travelling wave fronts, enabling the derivation of a relation between wave speed and the form of initial data. In the present paper, only large time behaviour is focused on, and only the equation given by linearizing about $u=0$ is solved. It is shown that this approach recovers (2), and moreover suggests that when $\xi>1$, there is a transition wave in $u_{x} / u$, moving ahead of the $u$ wave front at a faster speed.

\section{Solution by Laplace transforms}

Equation (1) is considered with initial data satisfying $u=O_{s}\left(\mathrm{e}^{-\xi x}\right)$ as $x \rightarrow \infty$. It is assumed that the solution evolves towards a travelling wave, whose speed $a$ remains to be determined: the calculation will confirm that $a$ is indeed given by the expression $c(\xi)$ in (2). For convenience, work will be carried out in terms of $t$ and the travelling wave coordinate $z=x$-at, rather than in terms of $t$ and $x$, so that the linearized equation is

$$
u_{t}=u_{z z}+a u_{z}+u
$$

Here, the subscripts $t$ and $z$ denote partial derivatives. This equation is considered on $0<z<\infty$, with end conditions

$$
\begin{array}{rll}
u_{z}+\xi_{0} u=0 & \text { at } & z=0 \\
u=O_{s}\left(\mathrm{e}^{-\xi z}\right) & \text { as } & z \rightarrow \infty \\
u=\mathrm{e}^{-\xi z} & \text { at } & t=0
\end{array}
$$


Conditions (4b) and (4c) reflect the initial conditions, while (4a) reflects the fact that all travelling wave solutions of the Fisher equation decay to $u=0$ exponentially. In fact, standard analysis of the travelling wave ordinary differential equations (ODEs) for (1) (Grindrod, 1991; Kolmogoroff et al., 1937) implies that the decay rate $\xi_{0}$ is related to the supposed wave speed $a$ by

$$
\xi_{0}=\frac{1}{2}\left[a-\sqrt{a^{2}-4}\right]
$$

Equation (3) is solved subject to (4) and (5) using the method of Laplace transforms. This is made easier by writing $w=u \cdot \exp \left[a z / 2+\left(a^{2} / 4-1\right) t\right], \alpha=\xi_{0}-a / 2$ and $\beta=\xi-a / 2$, giving

$$
\begin{array}{ll}
w_{t}=w_{z z} \text { subject to } & w_{z}+\alpha w=0 \text { at } z=0 \\
& w=O_{s}[\exp (-\beta z)] \text { as } z \rightarrow \infty \\
& w=\exp (-\beta z) \text { at } t=0
\end{array}
$$

Taking the Laplace transform with respect to time $t$ gives a simple ODE, with the following unique solution satisfying the boundary and initial conditions:

$$
W(z, s)=\frac{1}{2 \beta(\beta+\alpha)}\left[-\frac{\beta+\alpha}{\sqrt{s}-\beta}+\frac{\alpha-\beta}{\sqrt{s}+\beta}+\frac{2 \beta}{\sqrt{s}-\alpha}\right] \mathrm{e}^{-z \sqrt{s}}+\frac{\mathrm{e}^{-\beta z}}{s-\beta^{2}}
$$

Here a capital letter is used to denote the Laplace transform and $s$ is the transform variable. This can be inverted using standard transforms, to give

$$
\begin{aligned}
w(z, t) & =\frac{\exp \left[-z^{2} /(4 t)\right]}{2(\beta+\alpha)}\left[2 \alpha \mathscr{F}\left(-\alpha \sqrt{t}+\frac{z}{2 \sqrt{t}}\right)+(\beta+\alpha) \mathscr{F}\left(\beta \sqrt{t}-\frac{z}{2 \sqrt{t}}\right)\right. \\
& \left.+(\beta-\alpha) \mathscr{F}\left(\beta \sqrt{t}+\frac{z}{2 \sqrt{t}}\right)\right]
\end{aligned}
$$

where $\mathscr{F}(y) \equiv \exp \left(y^{2}\right) \operatorname{erfc}(y)$.

The value of $a$ is determined by the behaviour of $u(z=0, t)$ that is implied by (7). Substituting $z=0$ into (7) gives

$$
\begin{aligned}
w(z=0, t)= & \frac{\alpha}{\alpha+\beta} \mathscr{F}(-\alpha \sqrt{t})+\frac{\beta}{\alpha+\beta} \mathscr{F}(\beta \sqrt{t}) \\
& \sim \begin{cases}{[2 \beta /(\alpha+\beta)] \exp \left(\beta^{2} t\right) \mathscr{I}[\beta<0],} & \text { if } \alpha<0 \text { and } \beta \neq 0 \\
1 /(\beta \sqrt{\pi t})+2 \exp \left(\beta^{2} t\right) \mathscr{I}[\beta<0], & \text { if } \alpha=0 \text { and } \beta \neq 0 \\
-1 /(\alpha \sqrt{\pi t}), & \text { if } \alpha<0 \text { and } \beta=0 \\
1, & \text { if } \alpha=0 \text { and } \beta=0\end{cases}
\end{aligned}
$$

Here, standard asymptotic expansions are used for the complimentary error function, which imply that

$$
\mathscr{F}(y) \sim 1 /(y \sqrt{\pi})+2 \exp \left(y^{2}\right) \mathscr{I}[y \leqslant 0]
$$

as $y \rightarrow \infty$, where $\mathscr{I}$ denotes the indicator function defined by $\mathscr{I}$ [TRUE] $=1$, 
$\mathscr{I}[\mathrm{FALSE}]=0$. Note that (5) implies that $\alpha \leqslant 0$. In the case $\alpha=\beta=0$, (7) is not valid, but the solution of (6) is trivially $w \equiv 1$ in this case.

Under the assumption that the initial conditions (4c) evolve to travelling wave solutions in $u$, the value of $u(z=0, t)$ must approach a non-zero constant in the limit as $t \rightarrow \infty$. Since

$$
u(z=0, t)=\mathrm{e}^{\left(1-a^{2} / 4\right) t} w(z=0, t)=\mathrm{e}^{-\alpha^{2} t} w(z=0, t)
$$

this is possible only if $\alpha=\beta \leqslant 0$, i.e. $\xi_{0}=\xi \leqslant a / 2$. Together with (5), this implies $a=\xi+1 / \xi$ and $\xi \leqslant 1$, which is part of formula (2).

When $\xi>1$, there is no wave speed $a$ for which $\lim _{t \rightarrow \infty} u(z=0, t)$ is finite and non-zero. The relevant case then is in fact $\alpha=0, \beta>0$, i.e. $\xi_{0}=a / 2, \xi>a / 2$; using (5), this implies in turn that $a=2$. These calculations then give $u(z=0, t) \sim$ $1 /(\beta \sqrt{\pi t})$ as $t \rightarrow \infty$. Although this does tend to zero as $t \rightarrow \infty$, this approach is only algebraic and can be corrected. Specifically, consider the behaviour of the solution (7) at $z=-\frac{1}{2} \log t$ rather than $z=0$; even though (3) has been solved on $(0, \infty)$, the solution is well defined for negative values of $z$. This has no effect on the large time form for $w$, i.e. $w\left(z=-\frac{1}{2} \log t, t\right) \sim w(z=0, t)$ as $t \rightarrow \infty$. However, conversion to $u$ has the effect of multiplying the large time behaviour at $z=0$ by $t^{1 / 2}$; thus $u\left(z=-\frac{1}{2} \log t, t\right) \sim t^{1 / 2} u(z=0, t) \sim 1 /[\beta \sqrt{\pi}]$ as $t \rightarrow \infty$, a non-zero constant as required.

It is well known that the wave speed for the Fisher equation does indeed have a correction proportional to $-\log t / t$ for rapidly decaying initial data; this was first established by Bramson $(1978,1983)$. In fact, the nonlinear terms also make a contribution to this correction, and the actual wave speed is $2-3 \log t /(2 t)+\ldots$, rather than the $2-\log t /(2 t)+\ldots$ predicted by the linear analysis (Bramson, 1978, 1983); this difference is discussed in a more general setting by van Saarloos (1989). The slow convergence of the wave speed reflects a fundamentally different type of convergence to travelling wave solutions in the cases $\xi \leqslant 1$ and $\xi>1$. In the former case, the solution approaches the travelling wave uniformly in $x$. However, when $\xi>1$ the convergence is much weaker, with uniform convergence only in the shape of the solution. This distinction was first recognized by Stokes (1976).

\section{A transition in $u_{x} / u$}

The wave speed formula (2) has been known for many years. However, the derivation presented here, via explicit solution of the linearized equation in a moving frame, is new, and provides novel insights into the mechanism of wave speed selection. In particular, the solution enables detailed determination of the behaviour of $u_{x} / u$, and this application of (7) is now discussed. The ratio $u_{x} / u$ is of interest because it is clear that when $\xi>1$, there must be a transition in $u_{x} / u$ ahead of the $u$ wave, since $u_{x} / u \rightarrow-\xi$ as $x \rightarrow \infty$, while $u_{x} / u=-1$ in the tail of the $u$ wave of minimum speed. To investigate this, the behaviour of $u_{x} / u$ is considered as $t \rightarrow \infty$ with $z \sim 2 \gamma t$; here $\gamma$ is an arbitrary, strictly positive constant. When $\alpha=\beta \leqslant 0$ (corresponding to $\xi \leqslant 1$ ), it is straightforward to show that $u_{x} / u \rightarrow-\xi$ for all $\gamma$, as expected. However, for $\alpha=0, \beta>0$ (corresponding to $\xi>1$ ), the situation is more complicated. In this case

$$
w=\frac{1}{2} \mathrm{e}^{-\gamma^{2} t}[\mathscr{F}(\{\beta-\gamma\} \sqrt{t})+\mathscr{F}(\{\beta+\gamma\} \sqrt{t})]
$$




$$
\frac{u_{x}}{u}=\frac{u_{z}}{u}=\frac{w_{z}}{w}-\frac{a}{2}=\left.\frac{1}{2 t w} \frac{\partial w}{\partial \gamma}\right|_{t \text { constant }}-\frac{a}{2}
$$

and using the relation $\mathscr{F}^{\prime}(y)=2 y \mathscr{F}(y)-2 / \sqrt{\pi}$, this implies

$$
u_{x} / u=-\xi+\frac{2 \beta \mathscr{F}(\{\beta+\gamma\} \sqrt{t})}{\mathscr{F}(\{\beta-\gamma\} \sqrt{t})+\mathscr{F}(\{\beta+\gamma\} \sqrt{t})}
$$

Using the asymptotic behaviour for $\mathscr{F}$ given in (8) shows that as $t \rightarrow \infty$

$$
\mathscr{F}(\{\beta+\gamma\} \sqrt{t}) \sim 1 /[(\beta+\gamma) \sqrt{\pi t}]
$$

and

$$
\mathscr{F}(\{\beta-\gamma\} \sqrt{t}) \sim 1 /[(\beta-\gamma) \sqrt{\pi t}]+2 \exp \left\{(\beta-\gamma)^{2} t\right\} \mathscr{I}[\beta \leqslant \gamma]
$$

Thus, as $t \rightarrow \infty$ with $z \sim 2 \gamma t$

$$
u_{x} / u \sim \begin{cases}-\xi, & \text { if } \gamma>\beta \\ -\xi+\frac{2 \beta /(\beta+\gamma)}{1 /(\beta-\gamma)+1 /(\beta+\gamma)}=-a / 2-\gamma=-x / 2 t, & \text { if } \gamma<\beta\end{cases}
$$

Thus the solution for $u_{x} / u$ evolves to a transition wave, moving with absolute speed $2 \beta+a=2 \xi$; here the term 'absolute' means that this is the speed with respect to the spatial coordinate $x$ (rather than $z$ ). Note that this speed is greater than that of the $u$ wave, since $\xi>1$ and $a=2$. Ahead of this transition, $u_{x} / u \sim-\xi$, which is the decay rate imposed by the initial conditions, while behind it, $u_{x} / u \sim-x / 2 t$, which is the form corresponding to the fundamental solution of the diffusion equation, and arises from the diffusion of the initial data. Note that this latter expression implies that $u_{x} / u \sim-1$ in a frame moving with the wave speed $a=2$, as expected.

The identification of this transition in decay rates is, to the best of the author's knowledge, a new result. The transition is of course not a constant shape wave, since the limiting form behind the wave is a function of $t$; an example is illustrated in Fig. 1 , showing $u_{x} / u$ in a frame moving with speed $2 \xi$ at three successive times. In applications to biology and chemistry, reaction-diffusion models such as the Fisher-KPP equation are sometimes criticized on the basis that the diffusion term implies infinite speeds of propagation. The demonstration of a transition in decay rates shows that in the case of rapidly decaying initial data, the diffusion of this initial condition actually results in propagation at a finite speed, namely twice the initial decay rate.

\section{Application to reaction-diffusion systems}

In principle, the method presented here, namely linearization of the partial differential equation, solution via Laplace transforms and determination of large-time behaviour, could be applied to systems of several coupled reaction-diffusion equations. Numerical evidence indicates that in a number of such systems that are used in applications, the wave speed depends on initial data in a manner analogous to the Fisher-KPP equation (Dale et al., 1997; Manoranjan \& Mitchell, 1983; Sherratt, 1994); however, analytical analogues of the speed formula (2) exist only for a few special cases (Billingham \& Needham, 1992; Champneys et al., 1995; 


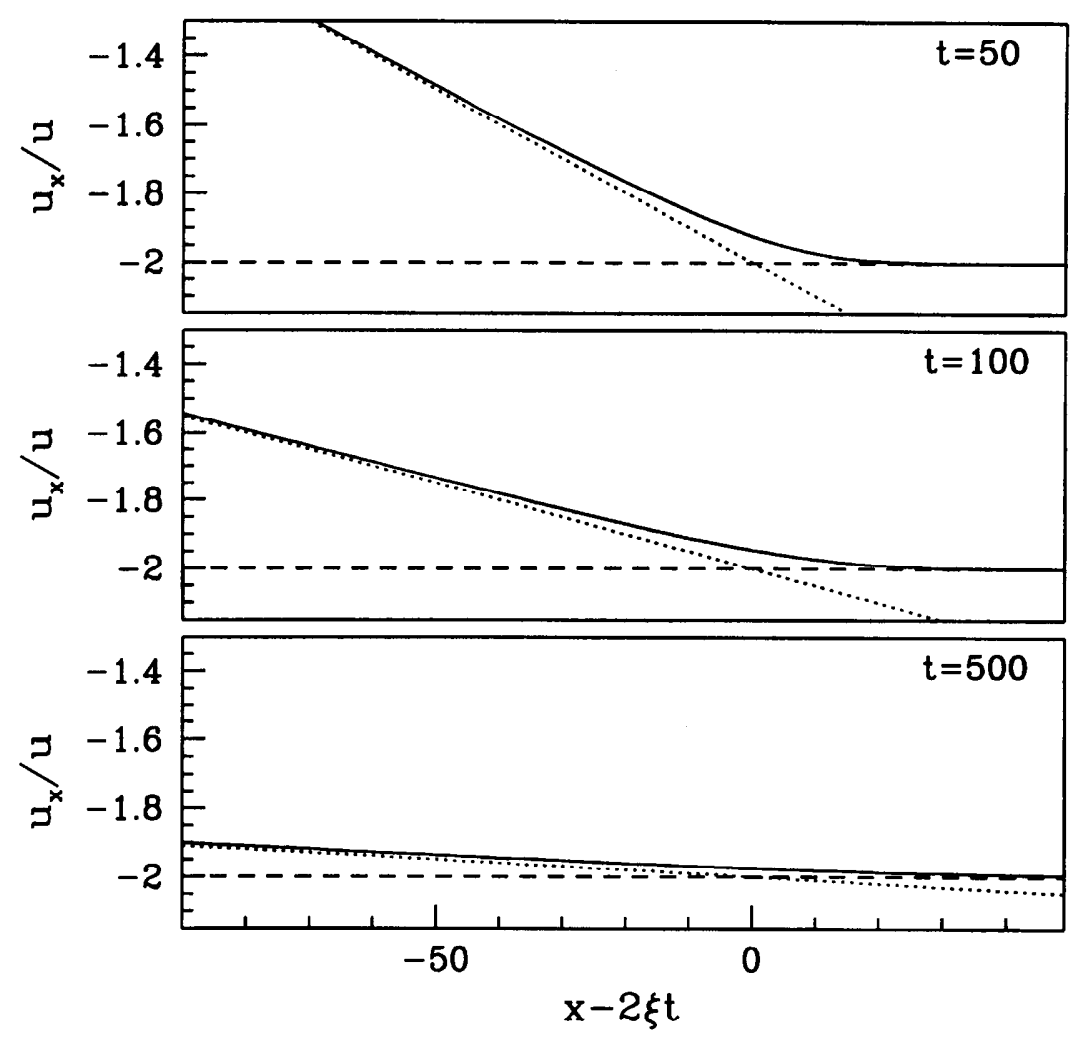

Fig. 1. An illustration of the large time form of $u_{x} / u$ ahead of the $u$ wave in the Fisher-KPP equation (1) when $\xi \leqslant 1$. The value of $u_{x} / u$, calculated from (9), is plotted as a function of the moving coordinate $x-2 \xi t$, at three successive times, for $\xi=2$; recall that $2 \xi$ is the speed of the transition in $u_{x} / u$. The dotted line is $-x / 2 t$, which is the form of $u_{x} / u$ behind the transition, and the dashed line denotes the value of $-\xi$, which is the value of $u_{x} / u$ ahead of the transition.

Freidlin, 1985, 1991). The transition from one to several equations would increase the algebraic complexity of the solution considerably, but poses no fundamental difficulties. The main problem is in fact that the equivalent of (5), which gives the relationship between the speed of a travelling wave solution and the decay rate of its tail, is not known for most reaction-diffusion systems. This is simply an ODE problem, but requires determination of the detailed form of a heteroclinic connection in the phase-space of the travelling wave ODEs, which is notoriously difficult in more than two dimensions.

A rare example in which the equivalent to (5) is known is the predator-prey model:

$$
\begin{gathered}
h_{t}=h(1-h-p) \\
p_{t}=p_{x x}+k_{1} p\left(h-k_{2}\right)
\end{gathered}
$$

Here $h(x, t)$ and $p(x, t)$ denote prey and predator densities, with $x$ a one-dimensional spatial coordinate and $t$ denoting time; $k_{1}$ and $k_{2}<1$ are positive constants (see May (1981) for a review of this and other related predator-prey models). Dunbar proved that for both (10) (Dunbar, 1983) and the corresponding model with nonzero prey diffusion (Dunbar, 1994), there is a travelling wave solution for all wave 
speeds greater than a critical minimum value; these waves represent the invasion of prey by predators, and have $h=1, p=0$ ahead of them, and $h, p \neq 0$ behind them. In the case of (10), Dunbar's proof (Dunbar, 1983) implies that the critical minimum wave speed is $2\left\{k_{1}\left(1-k_{2}\right)\right\}^{1 / 2}$, and that the waves decay to $h=1, p=0$ with an exponential decay rate $\frac{1}{2}\left[a-\left\{a^{2}-4 k_{1}\left(1-k_{2}\right)\right\}^{1 / 2}\right]$. Knowledge of this relationship between speed and decay rate enables the approach used in the present paper to be applied to (10). In fact, this is essentially trivial, since (10b) decouples from (10a) when one linearizes about $h=1, p=0$, so that the calculation is the same as that presented here. This suggests that the wave speed is given in terms of the initial decay rate $\xi$ by $\left[\xi+k_{1}\left(1-k_{2}\right) / \xi\right]$ if $\xi \leqslant \sqrt{k_{1}\left(1-k_{2}\right)}$, with the minimum speed selected for larger values of $\xi$ : this is the direct analogue of (2), and there is also a transition in $p_{x} / p$ and $h_{x} / h$, analogous to that illustrated in Fig. 1, moving ahead of the predator-prey wave front at a greater speed.

More generally, as new results on the detailed form of travelling wave solutions become available, the approach described here will enable prediction of the initial conditions that will generate these waves, and the corresponding dynamics of decay rates ahead of the wave fronts. This information is crucial for a wide range of applications, since the dependence of wave speeds on model parameters is often the most important prediction of reaction-diffusion systems. For example, a number of reaction-diffusion models have been proposed for the study of intracellular calcium signalling (Goldbeter et al., 1990; Sneyd et al., 1995); calcium is a central regulator of cell behaviour, and plays a key role in physiology and developmental biology. Detailed experimental data are available on the speed of calcium signalling waves in a range of conditions, and the comparison of this data with theoretical predictions is vital in the estimation of model parameters. Another prototype application is wound healing in the corneal epithelium (Dale et al., 1994). Here, detailed parameter estimation is possible using in vitro experimental data, so that an analytical prediction of wave speeds can be used to assess the ability of potential clinical therapies to increase the rate of repair. In both of these applications, approximate expressions for wave speeds are currently in use, and the derivation of exact wave speed formulae would be a very significant advance.

\section{Acknowledgements}

I am grateful to Steven Dunbar, Markus Owen and Pam Wiener for helpful discussions.

\section{References}

Billingham, J. and Needham, D. J. (1992) The development of travelling waves in quadratic and cubic autocatalysis with unequal diffusion rates. III: Large time development in quadratic autocatalysis. Quarterly of Applied Mathematics 50, 343-372.

Booty, M. R., Haberman, R. and Minzoni, A. A. (1993) The accommodation of travelling waves of Fisher's type to the dynamics of the leading tail. SIAM fournal of Applied Mathematics 53, 1009-1025.

Bramson, M. D. (1978) Maximal displacement of branching Brownian motion. Communications in Pure and Applied Mathematics 31, 531-581.

Bramson, M. D. (1983) Convergence of solutions of the Kolmogorov equation to travelling waves. Memoirs AMS 44 (285).

Champneys, A., Harris, S., Toland, J., Warren, J. and Williams, D. (1995) Algebra, analysis and probability for a coupled system of reaction-diffusion equations. Philosophical Transactions of the Royal Society London A 350, 69-112. 
Dale, P. D., Maini, P. K. and Sherratt, J. A. (1994) Mathematical modelling of corneal epithelial wound healing. Mathematical Biosciences 124, 127-147.

Dale, P. D., Sherratt, J. A. and Maini, P. K. (1997) The role of fibroblast migration in collagen fibre formation during foetal and adult dermal wound healing. Bulletin of Mathematical and Biology 59, 1077-1100.

Dunbar, S. R. (1983) Travelling wave solutions of diffusive Lotka-Volterra equations. fournal of Mathematical and Biology 17, 11-32.

Dunbar, S. R. (1994) Travelling wave solutions of diffusive Lotka-Volterra equations: a heteroclinic connection in $\mathrm{R}^{4}$. Transactions of AMS 286, 557-594.

Fisher, R. A. (1937) The wave of advance of advantageous genes. Annals of Eugenics 7, 353-369.

Freidlin, M. (1983) On wave front propagation in multicomponent media. Transactions of AMS 276, 181-191. See also the erratum, Transactions of $A M S$ 289, 429 (1985).

Freidlin, M. (1991) Coupled reaction-diffusion equations. Annals of Probability 19, 29-57.

Goldbeter, A., Dupont, G. and Berridge, M. J. (1990) Minimal model for signal-induced $\mathrm{Ca}^{2+}$ oscillations and for their frequency encoding through protein phosphorylation. PNAS USA 87, $1461-1465$.

Grindrod, P. (1991) Patterns and Waves (OUP, Oxford).

Holmes, E. E., Lewis, M. A., Banks, J. and Veit, R. R. (1994) Partial differential equations in ecology: spatial interactions and population dynamics. Ecology 75, 17-29.

Kolmogoroff, A., Petrovskii, I. and Piscounoff, N. (1937) Etude de l'équation de la diffusion avec croissance de la quantité de matière et son application à un problème biologique. Moscow University Bulletin of Mathematics 1, 1-25.

Larson, D. A. (1978) Transient bounds and time asymptotic behaviour of solutions of nonlinear equations of Fisher type. SLAM Yournal of Applied Mathematics 34, 93-103.

McKean, H. P. (1975) Application of Brownian motion to the equation of Kolmogorov-PetrovskiiPiscounoff. Communications in Pure and Applied Mathematics 28, 323-331.

Manoranjan, V. S. and Mitchell, A. R. (1983) A numerical study of the Belousov-Zhabotinskii reaction using Galerkin finite element methods. Fournal of Mathematical Biology 16, 251-260.

May, R. M. (1981) Stability and Complexity in Model Ecosystems (Princeton University Press, Princeton, New Jersey).

Murray, J. D. (1989) Mathematical Biology (Springer-Verlag, Berlin).

Needham, D. J., King, A. C. and Merkin, J. H. (1994) A reaction-diffusion model for autocatalytic polymerization: I. Permanent form travelling waves. IMA fournal of Applied Mathematics 53, 137-149.

Okubo, A., Maini, P. K., Williamson, M. H. and Murray, J. D. (1989) On the spatial spread of the grey squirrel in Britain. Proceedings of the Royal Society London B 238, 113-125.

Orme, M. E. and Chaplain, M. A. J. (1996) A mathematical model of vascular tumor growth and invasion. Mathematical Computational Modelling 23, 43-60.

Rothe, F. (1978) Convergence to travelling fronts in semilinear parabolic equations. Proceedings of the Royal Society of Edinburgh A 80, 213-234.

Saarloos, W. van (1989) Front propagation into unstable states. 2. Linear versus nonlinear marginal stability and rate of convergence. Physical Review A 39, 6367-6390.

Sherratt, J. A. and Murray, J. D. (1990) Models of epidermal wound healing. Proceedings of the Royal Society London B 241, 29-36.

Sherratt, J. A. (1994) On the evolution of periodic plane waves in reaction-diffusion systems of $\lambda-\omega$ type. SIAM fournal of Applied Mathematics 54, 1374-1385.

Sleeman, B. D. and Tuma, E. (1984) On exact solutions of a class of reaction diffusion equations. IMA fournal of Applied Mathematics 33, 153-168.

Sneyd, J., Keizer, J. and Sanderson, M. J. (1995) Mechanisms of calcium oscillations and waves: a quantitative analysis. FASEB fournal 9, 1463-1472.

Stokes, A. M. (1976) On two types of moving front in quasi-linear diffusion. Mathematical Biosciences 31, 307-315. 